\section{Efecto del enfoque de autorregulación del aprendizaje en la enseñanza de conceptos científicos en estudiantes universitarios en ciencias de la salud}

\author{
LUCÍA SANTELICES, CAROLINA WILLIAMS, \\ MAURICIO SOTO, ALBERTO DOUGNAC
}

\section{The efficiency of self-regulated learning in the teaching of scientific concepts to health sciences students}

Background: In health sciences, the predominant teaching methodology is traditional and emphasizes conveying knowledge. Nonetheless, new abilities must be taught now. This change shifts the prominence from professor to student and incorporates a concept called self-regulated learning, which involves the professor as a mediator and incorporates guidelines that facilitate learning diverse skills. Aim: To compare the effects of two teaching methodologies on the learning of key scientific concepts among health science students. Material and Methods: Two subgroups of equally complex concepts were randomly chosen to be taught either using traditional or self-regulation methodology. For the self-regulation methodology, two groups were formed. One learned only through self-regulation guidelines and the other learned through classes where the professor was a mediator. One hundred thirty seven freshman students from medicine, physical therapy, and nursing careers participated voluntarily in the study. Results: Self-regulation methodology impacted the learning process of scientific concepts in a positive way and showed significant differences with traditional teaching. The sole use of self-regulation guidelines alone generated similar results, compared to those obtained by students who attended lectures. Conclusions: Self-regulated learning would improve in learning efficiency and would reduce face-to-face class time.

(Rev Med Chile 2014; 142: 375-381)

Key words: Educational measurement; Learning; Teaching.

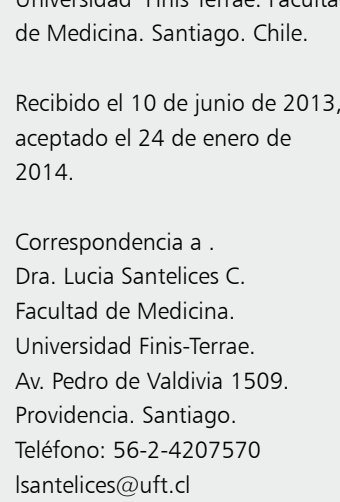

de Medicina Santiago Chile.

Recibido el 10 de junio de 2013 aceptado el 24 de enero de 2014

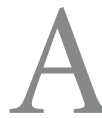

partir del enfoque de la enseñanza tradicional que se focaliza principalmente en la entrega de contenidos, la declaración de Bolonia $^{1}$ sugiere incorporar la enseñanza de habilidades en los procesos educativos tradicionales. En este contexto, se abre una discusión que plantea que,en las ciencias biomédicas, la metodología tradicional debe cambiarse por una metodología de enseñanza centrada en la comprensión del conocimiento disciplinar y en la utilización de éste, para que los estudiantes manejen la información y la utilicen correctamente en contextos profesio- nales diversos, resolviendo problemas prácticos ${ }^{2}$.

Preparar estudiantes como especialistas, expertos en un área disciplinar pero incapaces de aplicar y utilizar el conocimiento, versus preparar estudiantes que comprendan y utilicen correctamente los resultados del aprendizaje en la resolución de problemas profesionales, implica decidir dónde poner el énfasis en los procesos de enseñanza. Una enseñanza tradicional con clases expositivas, apoya la primera situación, en cambio, una enseñanza mediada en la cual el profesor junto con exponer, deja espacios para que el estudiante genere, inda- 
gue, responda interrogantes y proponga soluciones fundamentadas, apoya la segunda. Este último enfoque implica una metodología autorregulada que como veremos más adelante, exige al docente ceder su protagonismo y preparar a los estudiantes para aprender a aprender ${ }^{3}$.

La metodología autorregulada se entiende como un proceso en el cual el estudiante construye su propio sistema de aprendizaje. Para llevarlo a cabo, el docente planifica ejercicios de distintos niveles de comprensión: ejercicios que estimulen el recuerdo de información, la inferencia, la formulación de hipótesis y la evaluación ${ }^{4}$. Los estudiantes en forma autónoma identifican sus propias necesidades, establecen sus propios objetivos de aprendizajes para alcanzar la meta definida por el profesor, para lo cual es necesario que cada uno de ellos seleccione autónomamente los recursos que requiere para alcanzarlas ${ }^{5}$. Esta metodología docente permite al estudiante adquirir el desarrollo de habilidades de sistematización de la información, lo que facilita la comprensión de los conceptos centrales de la disciplina ${ }^{6}$. Al sistematizar estos conceptos se favorece la retención de los mismos en la memoria de largo plazo, lo que no ocurre con otras metodologías activas como por ejemplo el ABP (Aprendizaje Basado en Problemas) ${ }^{7}$. En esta última metodología docente los estudiantes también asumen un rol activo y movilizan sus recursos de aprendizajes, buscan y organizan información y la aplican para resolver problemas concretos, sin embargo, no sistematiza la información para preparar al estudiante para enfrentar el estudio independiente ni incorpora en su estructura el proceso de metacognición. En contraste, la autorregulación sistematiza el estudio independiente mediante un conjunto de preguntas y actividades que se inician con el recuerdo de información, la resolución de problemas y la generación de nuevas aplicaciones del conocimiento adquirido, que concluyen con ejercicios de carácter metacognitivo. Estos ejercicios estimulan al estudiante a descubrir sus propios vacíos en el aprendizaje y guía al estudiante para buscar autónomamente la información necesaria para superar esto vacíos.

Este cambio de enfoque ha generado discusión en el medio, dado que existe poca evidencia científica que demuestre en este ámbito la efectividad de los métodos de enseñanza autorregulados en ciencias de la salud.
En función de lo anterior, surge la interrogante: ¿qué tan válido y efectivo es enseñar conceptos científicos claves con una metodología autorregulada?

El presente estudio tiene como objetivo comparar resultados en el aprendizaje de conceptos científicos claves, al utilizar una metodología autorregulada vs una metodología tradicional.

\section{Metodología}

El presente estudio pretende comparar resultados de dos metodologías de enseñanza (tradicional y autorregulada) sobre la adquisición de conceptos científicos en un grupo de estudiantes de primer año de las carreras de Medicina, Kinesiología y Enfermería. De un universo de 230 estudiantes, 137 participaron de forma voluntaria, $(60 \%$ del universo). El 40\% restante se autoexcluyó del estudio debido a que el programa se dictó durante el mes de enero. Las dos metodologías fueron aplicadas en un curso de nivelación de 1 semana de duración con un total de $30 \mathrm{~h}$ cronológicas correspondientes a $40 \mathrm{~h}$ pedagógicas; $20 \mathrm{~h}$ pedagógicas se destinaron a la enseñanza tradicional y $20 \mathrm{~h}$ pedagógicas a la enseñanza con metodología de autorregulación.

El diseño del estudio contempló una selección de los conceptos científicos que debían ser entregados a los estudiantes en el curso, para alcanzar los objetivos de aprendizajes esperados. En el proceso de selección de dichos contenidos participó un equipo constituido por 6 docentes expertos en ciencias biológicas, quienes desarrollaron tres tareas:

- Analizar los contenidos científicos de los programas de estudio del primer semestre de cada carrera determinando el conjunto de conceptos claves de las materias allí contenidas.

- Analizar los programas oficiales de los cursos científicos de la enseñanza media.

- Realizar un análisis de discrepancia entre los conceptos aportados por la enseñanza media y los conceptos claves que requieren aprender los estudiantes en los primeros cursos universitarios.

Los 56 conceptos seleccionados (de complejidad equivalente) se distribuyeron aleatoriamente en dos subgrupos: 28 conceptos científicos A (CCA) y 28 conceptos científicos B (CCB). Los CCA fueron enseñados utilizando una metodo- 
logía autorregulada y los CCB fueron enseñados utilizando una metodología tradicional. Todos los estudiantes participaron de ambas metodologías. Hay que mencionar que en el caso de las intervenciones, tanto tradicional como autorregulada, el mismo equipo docente fue responsable de ambas modalidades de enseñanza.

Las clases diseñadas con metodología tradicional tuvieron como centro del proceso de enseñanza al docente, quien cumplió un rol protagónico en su papel de expositor. Por otro lado, las clases con metodología autorregulada tuvieron como protagonista al estudiante, desempeñando el docente sólo un rol mediador. En este caso, si bien se expusieron contenidos, se intercalaron actividades que implicaron síntesis, resolución de problemas utilizando los conceptos, creación de nuevas preguntas y resumen de conclusiones fundamentadas.

El método de autorregulación se puede concretar de dos formas, mediante una guía de autorregulación que estimula el desempeño independiente del estudiante o con clases mediadas por un profesor que guía las actividades de aprendizaje del estudiante de forma presencial. En este aspecto pareció interesante evaluar, cuál de las dos estrategias metodológicas de autorregulación tiene mayor impacto en la adquisición de conceptos científicos claves. De acuerdo a lo anterior, dentro de la metodología autorregulada se utilizaron las dos estrategias de aprendizaje, 46 estudiantes aprendieron los 28 CCA utilizando guías de autorregulación del aprendizaje y 91 estudiantes aprendieron los mismos 28 CCA sólo con la presencia de un profesor mediador (Figura 1). Esta distribución se originó cuando se les explicó a los estudiantes que existían estrategias autorreguladas para aprender el grupo de conceptos científicos A. Los estudiantes se inscribieron voluntariamente en una u otra modalidad.

Ambas metodologías (tradicional y autorregulada), se utilizaron en forma alternadas durante el mismo día. Para evitar en lo posible la contaminación entre metodologías se optó por:

1. Instruir a los profesores sobre la necesidad de mantenerse estrictamente apegados a la metodología docente predefinida.

2. Evidenciar el cumplimiento de la instrucción, a través de la supervisión de las clases por algunos de los autores utilizando una pauta de cotejo (LS y CW).

Para evaluar el impacto de ambas metodologías de enseñanza, se compararon los resultados iniciales de conocimientos científicos antes de la intervención con los resultados logrados después de un mes de realizada la intervención metodológica. Se diseñó un instrumento diagnóstico constituido por 56 preguntas de selección múltiple, cada pregunta refiere a un concepto clave el cual fue aplicado en calidad de pre-test y post-test ${ }^{8}$.

Los criterios de inclusión al estudio fueron los siguientes:

- Rendir prueba diagnóstica de conceptos científicos importantes para cursar las asignaturas del primer semestre (en calidad de pre-test).

- Asistencia a las clases del curso.

- Rendir prueba diagnóstica al finalizar el curso (en calidad de post-test).

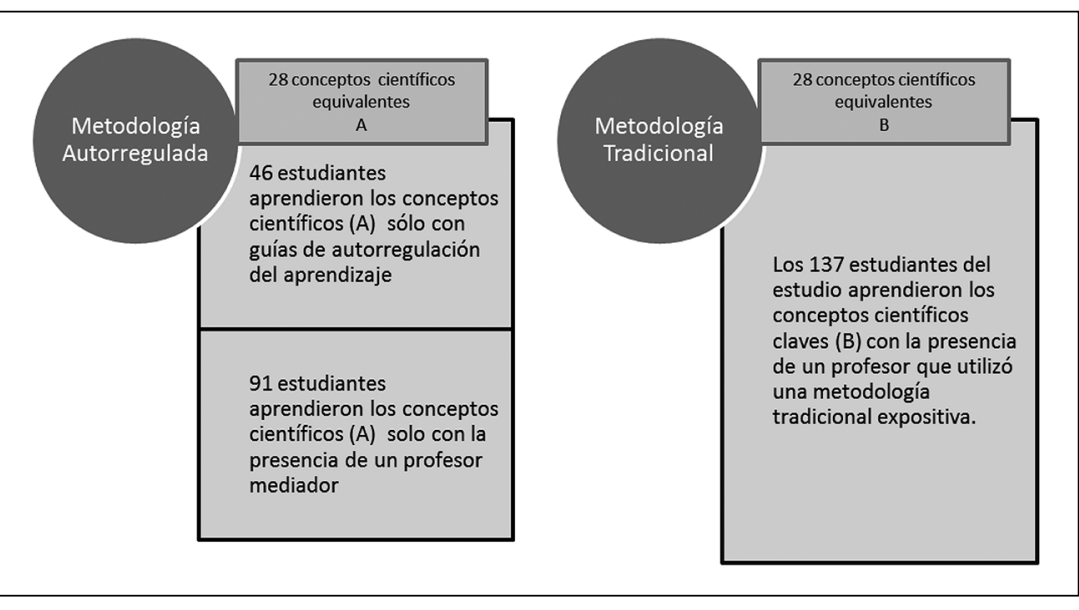

Figura 1. Diseño del estudio. 


\section{Análisis estadístico}

Para el análisis inferencial de los datos se realizaron los análisis de normalidad utilizando las pruebas de D-Agostino y Shapiro-Wilk a través del programa GraphPadPrism para un $\alpha$ de 0,05. Para comparar los resultados logrados por los estudiantes entre el pre-test y post-test se utilizó el test T-de Student. Se consideró significativo un $\mathrm{p}<0,05$.

\section{Resultados}

Como se mencionó anteriormente, la muestra quedó compuesta por 137 estudiantes de las carreras pertenecientes a la Facultad de Medicina de la Universidad Finis Terrae (Medicina: 56; Kinesiología: 33; y Enfermería: 48). Las edades de estos alumnos fluctuaron entre los 17 y 25 años con un promedio de 18,9 años. El mayor porcentaje de estudiantes provino de colegios particulares pagados, de nivel socioeconómico medio y sin presentar diferencias en la distribución por sexo.

De acuerdo al diseño del estudio, la totalidad de los alumnos (137) fueron sometidos a una metodología de enseñanza tradicional de los 28 conceptos científicos B seleccionados. Los mismos 137 estudiantes fueron sometidos a la metodología autorregulada con el propósito de enseñar los 28 conceptos científicos A, 46 de ellos lo hicieron a través de guías de autorregulación y los 91 restantes lo hicieron con un docente mediador (Figura 1).
Las características generales de la población estudiada y de sus subgrupos se muestran en la Tabla 1.

Para descartar posibles diferencias en los grados de dificultad entre los conceptos científicos A y $\mathrm{B}$, se compararon los resultados obtenidos por los estudiantes antes de la intervención (pre-test) no demostrándose diferencias estadísticamente significativas en los puntajes logrados por los alumnos, para ambos grupos de conceptos (Tabla 2).

Luego de efectuada la intervención se observó diferencias significativas en los puntajes obtenidos en el post test, cuyo objetivo fue evaluar el aprendizaje de conceptos científicos claves, dependiendo de la metodología utilizada.

La metodología autorregulada demostró favorecer el aprendizaje de conceptos científicos claves, respecto de la metodología tradicional. El número de respuestas correctas en el post test evidenció un mejor dominio de los CCA aprendidos en contraste con los CCB (Tabla 3). Llama la atención que no se evidencia aprendizaje con metodología tradicional cuando se comparan los puntajes obtenidos en el post test con los puntajes obtenidos en el pre test.

Al comparar los resultados obtenidos por los estudiantes que utilizaron la enseñanza autorregulada ya sea a través de guías o a través de un profesor mediador vs. enseñanza tradicional con un profesor expositor, se observa en ambos casos una diferencia significativa a favor de la metodología autorregulada (Tablas 4 y 5).

Tabla 1. Distribución estadística de la muestra según las variables intervinientes: edad, sexo, PSU y carreras

\begin{tabular}{|c|c|c|c|c|c|}
\hline Criterios & Indicadores & $\begin{array}{c}\text { Muestra total } \\
137\end{array}$ & $\begin{array}{c}\text { Tratamiento con } \\
\text { guías } \\
46\end{array}$ & $\begin{array}{c}\text { Tratamiento con } \\
\text { docente } \\
91\end{array}$ & Valor p \\
\hline Sexo & Mujeres (\%) & $62 \%$ & $28 \pm(61 \%)$ & $57 \pm(63 \%)$ & No significativo \\
\hline Edad & Media \pm DS & $18,6 \pm 1,7$ & $18,74 \pm 0,48$ & $18,59 \pm 1,00$ & No significativo \\
\hline NEM & Media \pm DS & $5,9 \pm 0,44$ & $5,99 \pm 0,44$ & $5,93 \pm 0,44$ & No significativo \\
\hline PSU & Media \pm DS & $624,1 \pm 62,3$ & $623 \pm 61,45$ & $625 \pm 64,52$ & No significativo \\
\hline Ciencias & Media \pm DS & $604,9 \pm 92,7$ & $600 \pm 98,02$ & $614 \pm 81,43$ & No significativo \\
\hline Medicina & \% participantes & $40 \%$ & $48 \%$ & $36 \%$ & No significativo \\
\hline Kinesiología & $\%$ participantes & $35 \%$ & $37 \%$ & $30 \%$ & No significativo \\
\hline Enfermería & \% participantes & $24 \%$ & $15 \%$ & $34 \%$ & No significativo \\
\hline
\end{tabular}


Finalmente, para comparar la efectividad de ambas metodologías de enseñanza autorreguladas (guía de autorregulación vs profesor mediador) se analizaron los puntajes obtenidos en ambas moda- lidades. No se encontraron diferencias estadísticamente significativas en los resultados alcanzados por los alumnos entre ambas metodologías de enseñanza (Tabla 6).

Tabla 2. Comparación de puntajes obtenidos en pre-test entre los conceptos científicos A (CCA) y conceptos científicos B (CCB)

\begin{tabular}{|lccc|}
\hline T de Student & $\begin{array}{c}\text { Ptje. Inicial CCA } \\
\mathbf{n = 1 3 7}\end{array}$ & $\begin{array}{c}\text { Ptje. Inicial CCB } \\
\mathbf{n = 1 3 7}\end{array}$ & Valor p \\
Prom \pm DS \pm SE & $21 \pm 8,5 \pm 0,68$ & $20 \pm 7,6 \pm 0,7$ & \\
(Ptje Min-Máx) & $(8-40)$ & $(9-43)$ & 0,7177 \\
Ptje. Percentil 75\% & 27 & 27 & \\
\hline
\end{tabular}

Tabla 3. Comparación entre los puntajes obtenidos en post test con metodología autorregulada (CCA) vs metodología tradicional (CCB)

\begin{tabular}{|lccc|}
\hline T de Student & $\begin{array}{c}\text { Ptje. Inicial CCA } \\
\mathbf{n = 1 3 7}\end{array}$ & $\begin{array}{c}\text { Ptje. Inicial CCB } \\
\mathbf{n = 1 3 7}\end{array}$ & Valor p \\
Prom $\pm \mathrm{DS} \pm \mathrm{SE}$ & $31 \pm 7,7 \pm 0,7$ & $20 \pm 7,2 \pm 0,7$ & \\
(Ptje Min-Máx) & $(20-52)$ & $(8-39)$ & $<0,0001 * * *$ \\
Ptje. Percentil 75\% & 39 & 25 & \\
\hline
\end{tabular}

Tabla 4. Comparación entre los puntajes obtenidos en post test con metodología autorregulada a través de Guías y metodología tradicional

\begin{tabular}{|lccc|}
\hline T de Student & $\begin{array}{c}\text { Ptje. Final CCA (con } \\
\text { guías) } \mathbf{n = 4 6}\end{array}$ & $\begin{array}{c}\text { Ptje. Final CCB } \\
\text { (tradicional) } \mathbf{n}=\mathbf{4 6}\end{array}$ & Valor $\mathbf{p}$ \\
\hline $\begin{array}{l}\text { Prom } \pm \text { DS } \pm \text { SE } \\
\text { (Ptje Min-Máx) }\end{array}$ & $33 \pm 7,8 \pm 1,2$ & $19 \pm 7,2 \pm 1,1$ & $<0,0001 * * *$ \\
Ptje. Percentil 75\% & $(22-50)$ & $(9-41)$ & \\
\hline
\end{tabular}

Tabla 5. Comparación entre los puntajes obtenidos en post test con metodología autorregulada a través de la conducción del docente como mediador y docente con metodología tradicional

\begin{tabular}{|lccc|}
\hline T de Student & $\begin{array}{c}\text { Ptje. Final CCA (con } \\
\text { docente) } \mathbf{n}=\mathbf{9 1}\end{array}$ & $\begin{array}{c}\text { Ptje. Final CCB } \\
\text { (tradicional) } \mathbf{n}=\mathbf{9 1}\end{array}$ & Valor p \\
Prom $\pm \mathrm{DS} \pm \mathrm{SE}$ & $31 \pm 7,6 \pm 0,7$ & $20 \pm 7,2 \pm 0,7$ & $<0,0001^{* * *}$ \\
(Ptje Min-Máx) & $(20-52)$ & $(8-39)$ & \\
Ptje. Percentil 75\% & 36 & 25 & \\
\hline
\end{tabular}

Tabla 6. Comparación entre los puntajes obtenidos en post test con metodología autorregulada a través de la aplicación de guías de autorregulación vs el docente como mediador

\begin{tabular}{|lccc|}
\hline T de Student & $\begin{array}{c}\text { Ptje. Final CCA (con } \\
\text { guías) } \mathbf{n}=\mathbf{4 6}\end{array}$ & $\begin{array}{c}\text { Ptje. Final CCA (con } \\
\text { docente) } \mathbf{n}=\mathbf{9 1}\end{array}$ & Valor $\mathbf{p}$ \\
\hline $\begin{array}{l}\text { Prom } \pm \text { DS } \pm \text { SE } \\
\text { (Ptje Min-Máx) }\end{array}$ & $33 \pm 7,8 \pm 1,2$ & $31 \pm 7,6 \pm 0,7$ & 0,083 \\
Ptje. Percentil 75\% & $(22-50)$ & $(20-52)$ & \\
\hline
\end{tabular}




\section{Comentarios finales}

En el enfoque tradicional de enseñanza de las ciencias, el papel protagónico lo tiene el docente, los estudiantes reciben pasivamente el conocimiento y lo memorizan sin ir, necesariamente, construyendo un sistema personal de aprendizaje. En este enfoque el docente es quien explica en forma directa los conceptos de una disciplina sin darse el tiempo de recoger los conocimientos de inicio que sus estudiantes podrían traer consigo, ni dejar espacios dentro de su exposición para reflexionar lo aprendido o recoger aportes que pudieran enriquecer el proceso enseñanza aprendizaje a través de breves discusiones.

Contrasta con lo anterior, el enfoque de autorregulación del aprendizaje, una nueva modalidad de enseñanza en la cual el papel protagónico lo tiene el alumno. El docente se orienta a estimular la autonomía del estudiante para enfrentar sus aprendizajes de manera guiada ${ }^{9}$. El profesor guía al estudiante para ordenar la información, explicarla, argumentar e interpretar la misma, hasta lograr formular hipótesis. Todas estas habilidades son esenciales para la comprensión profunda de los conocimientos científicos y base para la aplicación posterior del razonamiento clínico ${ }^{10}$. Esta nueva metodología permite al estudiante ir construyendo un sistema personal de aprender que le facilitará posteriormente la adquisición del método clínico ${ }^{11}$.

Por otro lado, la metodología tradicional en la enseñanza de las ciencias de la salud, ha mostrado limitaciones frente a las características de los nuevos estudiantes llamados "nativos digitales" 12 . Estos, tienen procesamiento múltiple de la información por lo que el discurso del docente les aburre, se distraen con facilidad y suelen lograr aprendizajes superficiales que les impiden aplicar el conocimiento a la solución de problemas ${ }^{13}$. Adicionalmente los nativos digitales tienen acceso rápido a la información pero carecen de criterios para discernir la validez de la misma ${ }^{12-14}$. Lo anterior, explicaría la ausencia de aprendizajes de $\mathrm{CCB}$, y además el posible olvido provocado por el aprendizaje superficial, que se demuestra con la disminución de los puntajes en el post test comparado con los puntajes logrados en el pre test. Este hecho justificaría la necesidad de cambiar la metodología tradicional por una metodología de tipo autorregulación del aprendizaje ${ }^{1}$.
Existen estudios que evidencian la efectividad de las metodologías de autorregulación del aprendizaje en la estimulación de habilidades relacionadas con el aprendizaje escolar de conceptos científicos en ciencias fácticas y ciencias sociales ${ }^{4,15,16}$, sin embargo, cuando se ha utilizado para generar autoinformes no ha demostrado su eficiencia ${ }^{17}$. Pero no existe evidencia de esta metodología aplicada al contexto de educación superior enciencias de la salud.

Los resultados sugieren que la metodología de autorregulación favorece la adquisición de conceptos científicos en los cursos iniciales de carreras de ciencias de la salud. Lo anterior se explica porque la autorregulación estimula habilidades para el manejo correcto de la información científica que incluye habilidades para organizar el conocimiento $y$ habilidades relacionadas con su aplicación, ya sea, mediante las actividades de una guía o mediante el rol mediador del profesor. Lo anterior, facilitará en el estudiante, el aprendizaje significativo de los conocimientos científicos y estimulará posteriormente el razonamiento clínico. Se ha observado empíricamente que durante el semestre los estudiantes siguen utilizando sus guías para solucionar problemas relacionados con los conceptos claves abordados en ellas. Además se observa que quienes aprendieron con autorregulación por guías o con profesor mediador recuerdan con más facilidad la información. Si bien los objetivos de este estudio no pretendían medir el logro de habilidades asociadas a la metodología de autorregulación, sería importante, su medición en un estudio posterior. Estudios realizados al respecto, no han demostrado asociación entre la preparación de las habilidades de los estudiantes y su desempeño académico ${ }^{18}$.

Por otra parte, existe evidencia que los alumnos que ingresan a primer año de universidad, no traen estrategias de estudio suficientemente desarrolladas para tener éxito académico y no son capaces de autorregular su comprensión ni su aprendizaje ${ }^{19}$. Por lo tanto, la incorporación de estrategias de autorregulación del aprendizaje por parte de un profesor mediador o por medio de guías de autorregulación, permite entregar en forma estructurada, lógica y secuencial diferentes conceptos científicos y facilitar su comprensión ${ }^{20}$. Además, son necesarias y altamente efectivas para estimular la autonomía de los estudiantes y entregarles una forma de organizar el conocimiento.

Nuestros resultados ponen en evidencia este 
hecho. La incorporación de nuevas metodologías docentes favorece el aprendizaje de conceptos científicos cuando estos además se enseñan en contextos significativos, es decir, asociados a requerimientos profesionales y resolución de problemas, que involucren al estudiante y lo obliguen a tomar decisiones y fundamentarlas.

Finalmente, nuestro estudio permitió comparar dos modalidades diferentes de metodología de autorregulación. El trabajo realizado con guías resultó tan efectivo como el trabajo del profesor mediador. Este aspecto sería importante de resaltar, ya que la utilización de este tipo de material, permitiría que el estudiante acceda a la información en forma autónoma y que el docente re-orientase su labor hacia lograr relacionar los saberes adquiridos con el contexto profesional en el que se desenvolverán en un futuro. El uso de guías permitiría además, contar con mayor número de horas docentes presenciales, que podrían emplearse para trabajar con más detalle y profundizar otras habilidades esenciales para desempeñarse efectivamente en las áreas de las ciencias de la salud ${ }^{9}$, tales como la comunicación efectiva, el trabajo en equipo, la investigación y actualización autónoma de los estudiantes en la literatura especializada, entre otras.

En nuestro estudio la utilización de metodologías orientadas al enfoque de autorregulación del aprendizaje resultó ser más efectiva que la enseñanza tradicional. Por lo anterior, pensamos que sería importante promover un cambio drástico en las estrategias de enseñanza que se utilizan en los primeros años de estudio de las carreras de la salud.

\section{Referencias}

1. Espacio Europeo de Enseñanza Superior. Declaración de Bolonia: Declaración conjunta de ministros europeos de enseñanza. 1999. Bolonia.

2. Arata A, Rodríguez E. Desafíos y perspectivas de la dirección estratégicas de las instituciones universitarias. Santiago, Chile: LOM, 2009.

3. Jorbá J, San Martí N. La regulación y autorregulación de los aprendizajes. Barcelona: Síntesis, 1997.

4. Gagliardi R. Los conceptos estructurantes en el aprendizaje por investigación. Barcelona: Enseñanza de las ciencias 1986; 4: 30-5.

5. Parra P, Pérez C, Ortíz L, Fasce E. El aprendizaje auto- dirigido en el contexto de la educación médica. Revista Educación en Ciencias de la Salud 2010; 7: 146-51.

6. Zimmerman J, Shunck DS. Self-regulated learning and achievement: theoretcal perspectives. New York: Mahwah, 2002. págs. 125-15.

7. Barell J. El aprendizaje Basado en problemas. Edit. Manantial. Buenos Aires. (1999).

8. Santelices L, Williams C, Zarate A, Soto M, Jara N, Dougnac A. Impacto de un programa de nivelación de ciencias básicas en estudiantes de primer año de la carrera de Medicina. Santiago, Chile: Rev Med Chile 2013; Vol. En proceso editorial.

9. Jorbá J, Casellas E. La Regulación y Auto regulación de los Aprendizajes, Editorial Síntesis 2004, Madrid, España.

10. Rodríguez LA, Valdez de la Rosa C, Salellas J. La adquisición de habilidades de razonamiento clínico en estudiantes de la carrera de Medicina. La Habana: Humanidades médicas 2013; 13: 72-87.

11. García Núñez RD. El método clínico en la atención primaria de salud: algunas reflexiones. 2010, Medisur (serie en internet) recuperado el 8 octubre de 2010 en: medisur.sld.cu/index.php/medisur/article/viewArticle/1323/372.

12. Gil D. Relaciones entre conocimiento escolar y conocimiento científico. Revista de Investigación en la Escuela 1994; 23: 17-32.

13. Morduchowicz R. La generación Multimedia. Buenos Aires: PAIDOS SAICF, 2008, Vol. Varios Capítulos.

14. Carlino P. Escribir, leer y aprender en la universidad. Fondo de Cultura Económica. Buenos Aires, 2005.

15. Nolen S, Haladyna TN. Motivation and studying in high school science. JRST 1990; 27: 115-26.

16. Pozo J, Gómez Crespo MA. Aprender a enseñar ciencias, Editorial Morata, Madrid, España. 2006.

17. Núñez JC, Solano P, González-Pineda J, Rosario P. Evaluación de los procesos de autorregulación mediante autoinforme. Psicothema 2006; 18: 353-8.

18. Zachariah MD, Huynh D, Charmaine R, Sturpe D, Kiser K. Readiness for Self-directed Learning and Academic Perfomance in an Abilities Laboratory Course. Am J Pharm Educ 2011; 10; 75 (2): 25.

19. Allgood WP, Risko VJ, Alvarez MC, Fairbanks MM. Factors that influence study. enR.F.Flippo y D.C. Caverly. Handbook of college Reading and study strategy research, NJ: LEA. 2000; 201-9.

20. García T, Pintrich PR. Regulating motivation and cognition in the classroom: The role of self-schemas and selfregulatiry strategies. En D.H. Schunk y B.J. Zimmerman (Eds.): Self-regulation of learning and performance. Issues and educational applications. Hillsdale, NJ: LEA. 1994. 